\title{
CUBIC NEUTRONS
}

\author{
FELIPE J. LLANES-ESTRADA* and GASPAR MORENO NAVARRO \\ Departamento de Física Teórica I, Univ. Complutense de Madrid, 28040 Madrid, Spain \\ *fllanes@fis.ucm.es
}

Received 28 October 2011

Revised 11 January 2012

Published 17 February 2012

\begin{abstract}
The neutron is largely spherical and incompressible in atomic nuclei. These two properties are however challenged in the extreme pressure environment of a neutron star. Our variational computation within the Cornell model of Coulomb gauge QCD shows that the neutron (and also the $\Delta^{3 / 2}$ baryon) can adopt cubic symmetry at an energy cost of about $150 \mathrm{MeV}$. Balancing this with the free energy gained by tighter neutron packing, we expose the possible softening of the equation of state of neutron matter.
\end{abstract}

Keywords: Hadron deformation; neutron stars; equation of state of neutron matter.

PACS Nos.: 26.60.-c, 14.20.Dh

The atomic nucleus is a close-packed system analogous to a cold quantum liquid. Its hadronic components are very incompressible, as revealed by the nuclear radius scaling with the mass number as $r_{A} \simeq r_{0} A^{1 / 3}$, with unit of length $r_{0} \simeq 1.2 \mathrm{fm}$. Nucleons there are quite optimally packed, as the (charge) nucleon radius has been precisely determined ${ }^{10}$ in electron scattering, and is approximately $0.88 \mathrm{fm}$. If we (grossly) think of the nucleon as a solid sphere, $74 \%$ of the nuclear volume is occupied, near the Keplerian limit ${ }^{8}$ reached by hcp or fcc lattices.

In neutron stars, gravity-compressed nuclear matter finds extreme pressure environments that change some of its properties. Recently, ${ }^{3}$ a two-solar mass neutron star has been discovered that takes nuclear physics to the limit (and we have recently proposed $^{4}$ that this is so much so as to constrain gravity for strong field intensity). At high pressure and density in the core of the star many exotic phases of hadron matter have been proposed, including a neutron superconductor/superfluid, various meson condensates, quarkyonic or Color-Flavor-Locked condensates, etc. Some of them have already been ruled out by the new, superheavy neutron star (see plot 3 in Ref. 12). 
At the crust one expects a thin atomic sheet ${ }^{14}$ followed by neutron-rich nuclear matter, and finally almost pure neutron matter (with a small number of protons in $\beta$-equilibrium). ${ }^{17}$

One intermediate phase that has long been proposed ${ }^{2}$ is a neutron crystal. In analogy with condensed matter systems (saliently ${ }^{3} \mathrm{He}$, a quantum liquid through $T=0$ that solidifies upon compression) neutron matter is assumed to locally adopt a periodic lattice-like arrangement to maximize density.

Our new observation is that when the environment loses its isotropy, the nucleon (largely spherical at rest) is subject to directional stresses and may deform to minimize the evacuated volume. Thus, for mass-energy densities $\varepsilon$ larger than $140 \mathrm{MeV} / \mathrm{fm}^{3} \simeq 3 m_{\pi}^{4}$, neutrons can no longer be considered pointlike objects subject to the rules of local, rotationally-invariant quantum field theory as elementary fields. Their composition and structure should start being taken into account, perhaps revisiting some of the hypotheses underlying the nuclear many-body problem approach based on QFT methods (particularly, rotational invariance).

We report an estimate of the deformability of the neutron with the renowned Cornell Hamiltonian

$$
\begin{aligned}
\mathcal{H}(\mathbf{x})= & \Psi^{\dagger}(\mathbf{x})(-i \boldsymbol{\alpha} \cdot \nabla+m \beta) \Psi \\
& +\frac{1}{2} \int \mathrm{d}^{3} y \Psi^{\dagger}(\mathbf{x}) T^{a} \Psi(\mathbf{x}) V(\mathbf{x}-\mathbf{y}) \Psi^{\dagger}(\mathbf{y}) T^{a} \Psi(\mathbf{y})
\end{aligned}
$$

a field theory model of Quantum Chromodynamics, tractable by many-body methods common in nuclear and condensed matter theory. The relativistic quark fields $\Psi$ interact via the color charge density $\Psi^{\dagger}(\mathbf{x}) T^{a} \Psi(\mathbf{x})$. The model has been amply exploited to treat the heavy quarkonium spectrum, ${ }^{6,16}$ as well as both light mesons and baryons ${ }^{13,1}$ with the Cornell potential $V(r)=\sigma r-\frac{4 \alpha_{s}}{3 r}$.

The model ground state $\left({ }^{3} P_{0}\right.$ quark-antiquark condensed vacuum) is treated in BCS approximation to generate constituent quarks from the current $m=5 \mathrm{MeV}$ quarks in the Hamiltonian, then the second-quantized wave function appropriate for three-quark baryons in terms of Bogoliubov-rotated quark creation operators $B^{\dagger}$ is

$$
|N\rangle=\frac{\epsilon^{i j k}}{6} F_{N}^{\lambda_{1} \lambda_{2} \lambda_{3}}\left(\mathbf{k}_{1}, \mathbf{k}_{2}, \mathbf{k}_{3}\right) B_{\mathbf{k}_{1} \lambda_{1} i}^{+} B_{\mathbf{k}_{2} \lambda_{2} j}^{+} B_{\mathbf{k}_{3} \lambda_{3} k}^{+}|\Omega\rangle .
$$

To approach lowest-lying baryons by the Rayleigh-Ritz variational principle, we employ the separable wave function ( $\rho, \lambda$ being three-body Jacobi coordinates with Cartesian components $\rho_{x}, \lambda_{z}$, etc.)

$$
F_{N}\left(\mathbf{k}_{\rho}, \mathbf{k}_{\lambda}\right)=\psi_{N}\left(\frac{\mathbf{k}_{\rho}}{\alpha_{\rho}}\right) \psi_{N}\left(\frac{\mathbf{k}_{\lambda}}{\alpha_{\lambda}}\right) .
$$

To choose a convenient variational wave function, we observe that the graph of

$$
\left(k_{x}^{N}+k_{y}^{N}+k_{z}^{N}\right)^{\frac{1}{N}}=1
$$



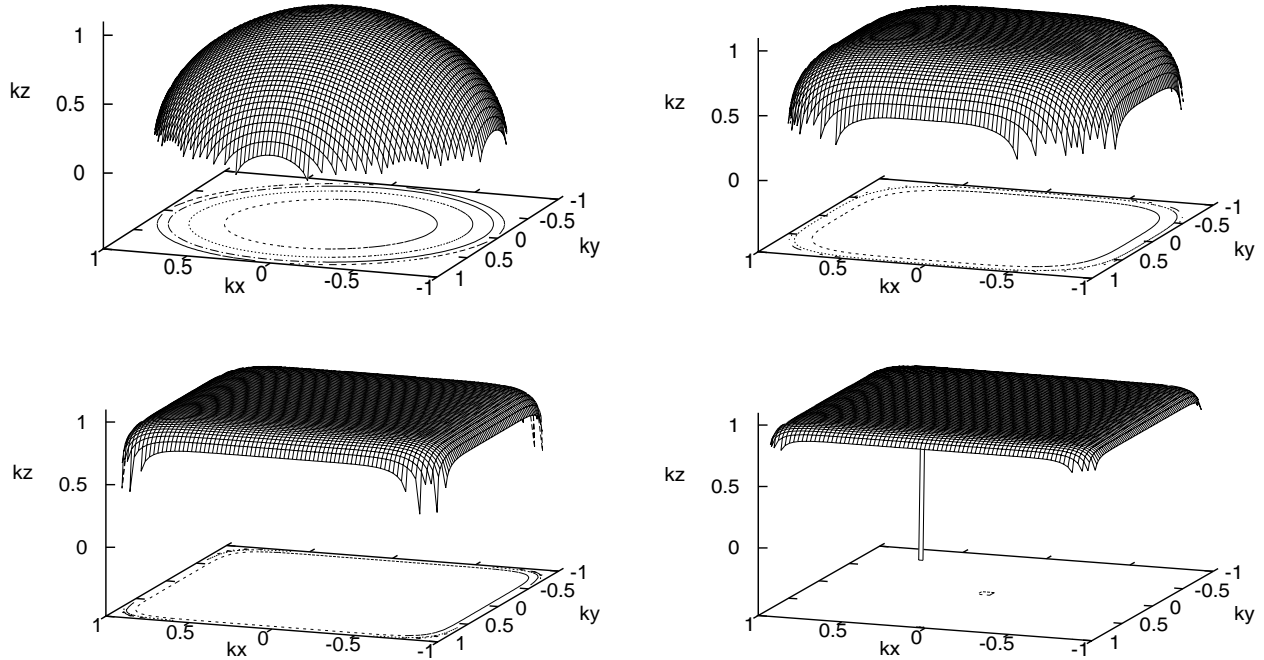

Fig. 1. Simple wave function shape in Eq. (4) that interpolates between sphere (for $N=2$ ), and cube $($ as $N \rightarrow \infty$ ) for $N=2,4,8,12$.

which is depicted in Fig. 1 for even $N$, interpolates between spherical symmetry for $N=2$ and octahedral (the cube's) symmetry, reached as $N \rightarrow \infty$. Therefore one can take an appropriately normalized $\psi_{N}$ from a precise numerical solution of the two-body problem and use

$$
F_{N}\left(\mathbf{k}_{\rho}, \mathbf{k}_{\lambda}\right)=\psi_{N}\left(\left(k_{\rho x}^{N}+k_{\rho y}^{N}+k_{\rho z}^{N}\right)^{\frac{1}{N}}\right) \psi_{N}\left(\left(k_{\lambda x}^{N}+k_{\lambda y}^{N}+k_{\lambda z}^{N}\right)^{\frac{1}{N}}\right)
$$

to study the three-quark system transiting between both symmetry groups.

The Hamiltonian expectation value $\langle N|H| N\rangle$ is a function of the two variational parameters $\alpha_{\rho}, \alpha_{\lambda}$, and is minimized with respect to them to find the best approximation to the neutron mass within this family of functions for $N=2$. To compute the matrix elements, we perform all relativistic spinor sums numerically, and the three-body nine-dimensional integral by Monte Carlo methods. ${ }^{7}$ The model approximation to the nucleon mass is $980 \mathrm{MeV}$ (with a Monte Carlo error of $40 \mathrm{MeV}$ ), the physical mass being $940 \mathrm{MeV}$. Should higher precision be necessary, one should resort to lattice gauge theory. ${ }^{5}$ This is not so for our purposes and we proceed to change the wave function symmetry, repeating the minimization for varying $N$.

We find that the neutron mass increases by about $150 \mathrm{MeV}$ between $N=2$ and $N=18$ (the last already very close to a cubic neutron), as depicted in Fig. 2 .

As a check of the spin-independence of our result, we have repeated the calculation for the $\Delta(1232)$ baryon, where all three quark spins are parallel, and obtained a ground state mass of 1250(50) $\mathrm{MeV}$.

The energy of shape-excitation is similar to the neutron case and reported in Fig. 3. This calculation also allows us to maintain that the point at $N=15$ in Fig. 2 was probably a fluctuation in the Monte Carlo program. Future, more precise calculations will eliminate this uncertainty. 


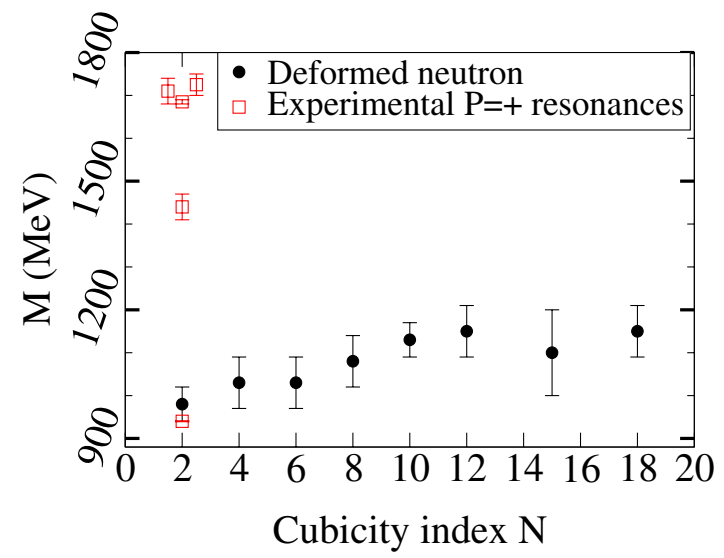

Fig. 2. Open squares: experimental neutron mass and known positive parity resonance excitations. ${ }^{15}$ Filled circles: variational minimum for the nucleon mass with the wave function in Eq. (3). At a cost of $50 \mathrm{MeV}$ the neutron already loses spherical symmetry for $N=4$, adopting an almost totally cubic shape for $150 \mathrm{MeV}$.

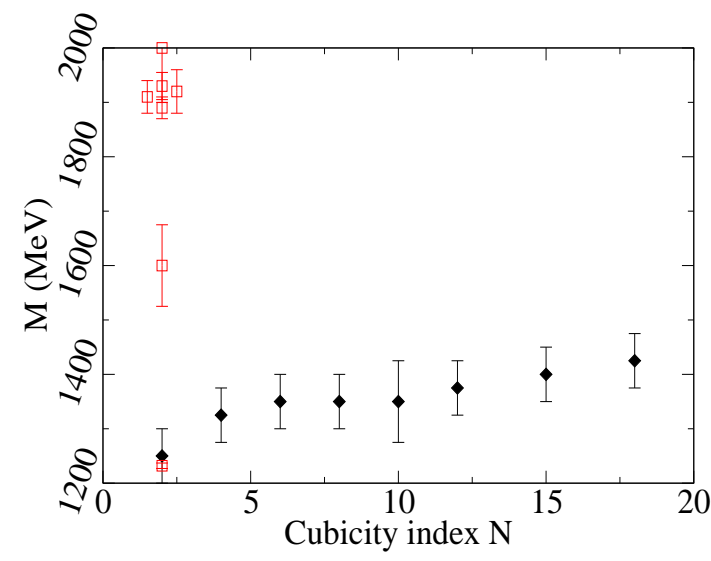

Fig. 3. Open squares: experimental $\Delta$-baryon mass and known positive parity resonance excitations. ${ }^{15}$ Filled circles: variational minimum for the $\Delta$ mass with the wave function in Eq. (3). The excitation cost to lose spherical symmetry is similar to that reported in Fig. 2 for the neutron.

We can offer the following heuristic argument supporting these numerical findings. If one takes a high $N$ (close to cubic) variational wave function, and performs a spherical harmonic analysis, $75 \%$ of the probability resides in the $Y_{00}$ spherically symmetric state, and an additional $24 \%$ in one of the $d$-waves $Y_{2 m}$ (mostly $m=0$ ) states. Weighing the experimental masses (several positive-parity excitations of the nucleon are also shown in Fig. 2) of ground $J^{P}=\frac{1}{2}^{+}(940 \mathrm{MeV})$ and $d$-wave excited $J^{P}=\frac{5}{2}^{+}(1680 \mathrm{MeV})$ nucleons in the proportion 3:1 yields $1125 \mathrm{MeV}$. This entails a cost of about $180 \mathrm{MeV}$ to change the neutron symmetry from spherical to cubic. 


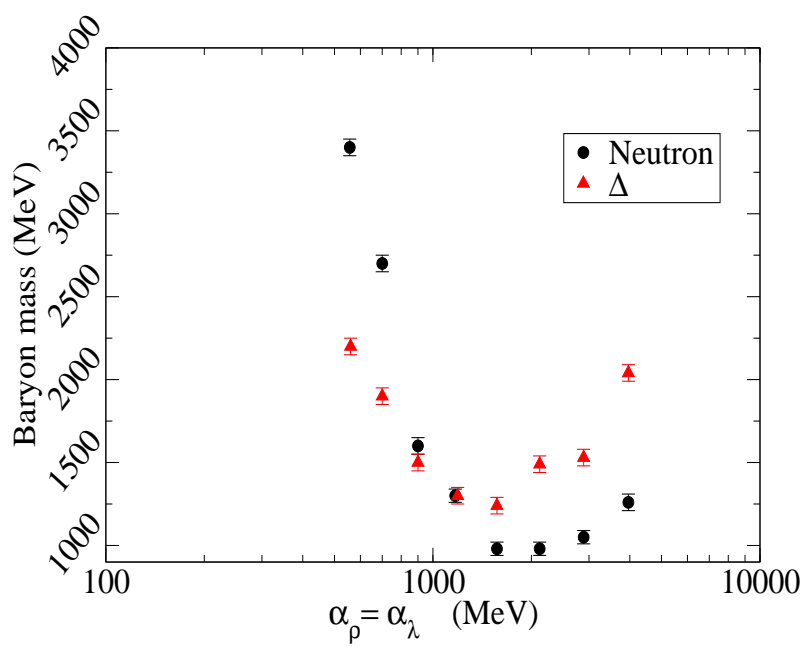

Fig. 4. Mass of nucleon and $\Delta$-baryon as function of only one variational parameter $\alpha_{\rho}=\alpha_{\lambda}$. In addition to a minimum corresponding to the best Rayleigh-Ritz representation of the particles within the function family, the energy cost of compression or dilatation of the wave function (at constant shape) can be appreciated.

Thus, from knowledge of the experimental spectrum and symmetry considerations alone one can already obtain the approximate answer. The same reasoning applies to the $150 \mathrm{MeV}$ excitation energy of the $\Delta$, since the relevant $d$-wave resonance energy is tagged by the $\Delta^{5 / 2+}(1900)$ and the $\Delta^{7 / 2+}(1950)$.

Although deformability at constant hadron volume, and not compressibility of the hadron, was the goal of our investigation, we can provide a feeling for the latter by setting both Jacobi scales equal $\alpha_{\rho}=\alpha_{\lambda}$ and studying the mass as function of this variational parameter alone. We refer the reader to Fig. 4. A variational minimum is clearly seen for both nucleon and $\Delta$, corresponding to their best RayleighRitz representation. Compressing or dilating the neutron can be faked by varying this scale, and the cost of doing so is evident in the figure, and of the order of magnitude that one naively expects. The neutron seems to be somewhat stiffer to momentum-space compression (position-space dilatation), while both particles react similarly to momentum-space dilatation (position-space compression).

The excitation energy that we have found is almost equal to that necessary to produce hyperons, since the lightest strange particle, $\Lambda$, has a mass of about $1115 \mathrm{MeV}$. Thus, if the nucleon's Fermi momentum is high enough to relax some of the $d$ quarks to $s$ quarks, it is also high enough to deform the nucleon, and both phenomena appear simultaneously. Their interplay is beyond our current undertaking.

At high enough pressure, the $150 \mathrm{MeV}$ energetic cost to deform the neutron can be provided by the Helmholtz free energy $P \Delta V$ obtained upon reducing the interstitial volume between neutrons, thanks to the cubic shape. The maximum gain as $N \rightarrow \infty$ in an fcc lattice, with packing fraction 0.74 , is $26 \%$ of the initial volume 


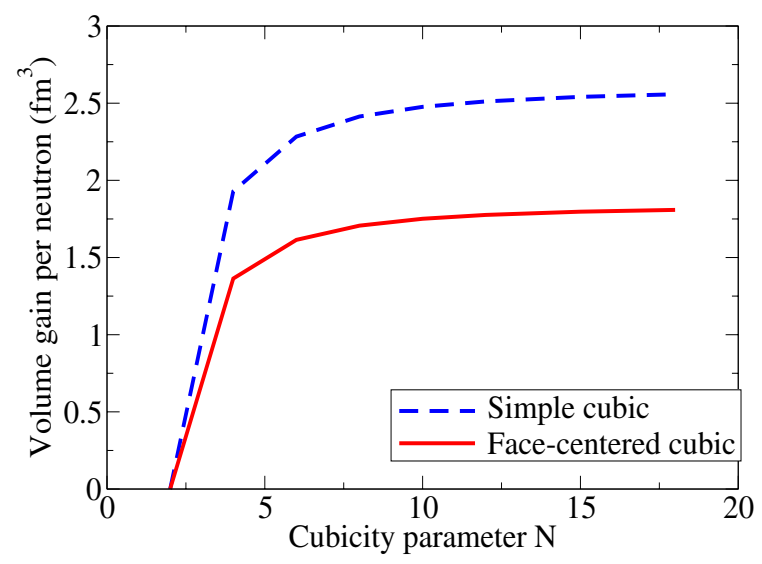

Fig. 5. Interstitial volume gain $\Delta V$ by deformation of the neutron wave function at constant neutron volume for two cubic lattices. The gain is naturally smaller for the more packing-efficient face-centered-cubic lattice, but still significant.

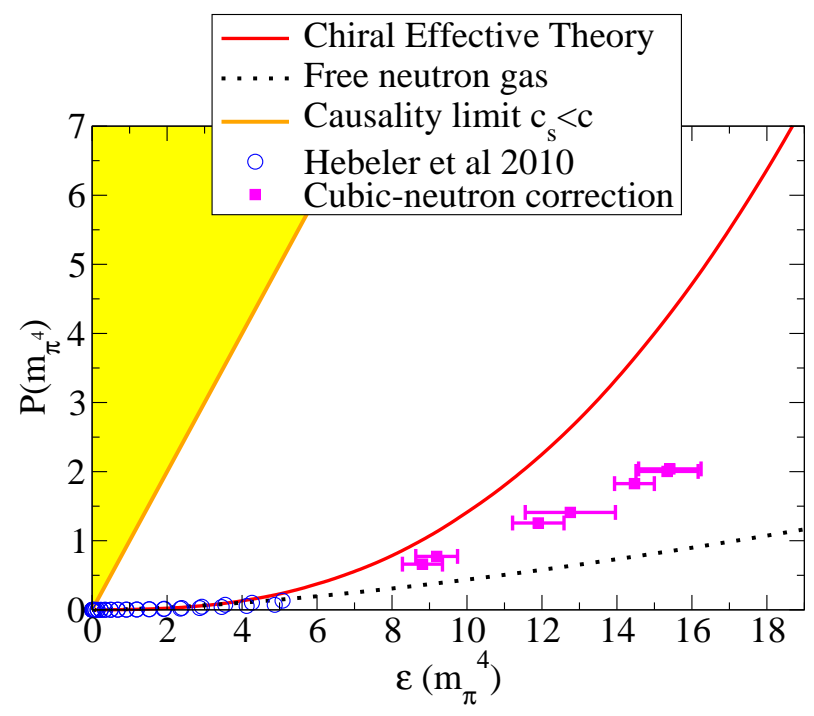

Fig. 6. (color online) Effect on the state of the art equation of state ${ }^{11,9}$ of neutron matter of the deformability of the neutron. The entire interval shown is relevant for neutron star structure. Nucleon deformability produces a softening of the equation of state additional to other known sources.

occupied by a spherical neutron. Estimating this volume for arbitrary $N$ through simple three-dimensional integration (see Fig. 5) we can, at fixed pressure, obtain the consequent increase in density. We plot in Fig. 6 the effect on the equation of state of neutron matter of the neutron deformability that we have found. The equation of state becomes softer (smaller pressure at given density) at densities relevant for neutron star structure. 
To conclude, let us observe that the bulk properties of neutron matter in the star will be affected by the neutrons adopting cubic symmetry (and patches of neutron matter becoming crystalline), particularly the Young modulus, the moment of inertia, etc. and this will surely provide detailed tests for future work. For example, certain types of seismic waves do not propagate in amorphous materials but do so in crystalline materials where there are elastic restoration forces. We are planning to analyze long-wavelength mechanical distortions of the star to see how the various phases (crystalline or fluid) interplay to produce crustal distortions, that will possibly be observable in the future.

\section{Acknowledgments}

F.J.L.E. thanks a Caja Madrid fellowship for advanced study and the hospitality of the theory group at TU-Munich and the Exzellenzcluster Origin and Structure of the Universe. This work has been supported by grants 227431HadronPhysics2 (EU), Consolider-CSD2007-00042, AIC10-D-000582, FPA200800592, FIS2008-01323, and UCM-BSCH GR58/08 910309 (Spain).

\section{References}

1. P. Bicudo et al., Phys. Rev. Lett. 103, 092003 (2009).

2. V. Canuto and S. M. Chitre, Nature Phys. Sci. 243, 63 (1973).

3. P. Demorest et al., Nature 467, 1081 (2010).

4. A. Dobado, F. J. Llanes-Estrada and J. A. Oller, arXiv:1107.5707.

5. S. Durr et al., Science 322, 1224 (2008).

6. E. Eichten, K. Gottfried, T. Kinoshita, K. D. Lane and T.-M. Yan, Phys. Rev. D 17, 3090 (1978).

7. T. Hahn, Comput. Phys. Commun. 168, 78 (2005).

8. T. C. Hales, J. Comput. Appl. Math. 44, 41 (1992).

9. K. Hebeler, J. M. Lattimer, C. J. Pethick and A. Schwenk, Phys. Rev. Lett. 105, $161102(2010)$.

10. R. J. Hill and G. Paz, Phys. Rev. D 82, 113005 (2010).

11. A. Lacour, J. A. Oller and U.-G. Meissner, Ann. Phys. 326, 241 (2011).

12. J. M. Lattimer and M. Prakash, arXiv:1012.3208.

13. F. J. Llanes-Estrada and S. R. Cotanch, Phys. Rev. Lett. 84, 1102 (2000).

14. F. J. Llanes-Estrada and G. M. Navarro, to appear in J. Astrophys. Space Sci.

15. K. Nakamura et al., J. Phys. G: Nucl. Part. Phys. 37, 075021 (2010).

16. J. M. Torres-Rincon and F. J. Llanes-Estrada, Phys. Rev. Lett. 105, 022003 (2010).

17. Ya. B. Zeldovich and I. D. Novikov, Stars and Relativity (Dover, 2011). 
Copyright of Modern Physics Letters A is the property of World Scientific Publishing Company and its content may not be copied or emailed to multiple sites or posted to a listserv without the copyright holder's express written permission. However, users may print, download, or email articles for individual use. 